Our study did not evidence whole-brain volume deficits in firstepisode bipolar disorder compared with healthy controls. This may indicate that a progressive decrease of whole-brain volume occurs over the course of the disease, and might be detectable only when multi-episode or chronic cases are considered. This is confirmed by the correlation found between gray matter loss and duration of illness in the meta-regression performed by Arnone et $a l^{1}$ and by the results of longitudinal studies demonstrating gray matter volume loss over time in the prefrontal cortex in young adults with bipolar disorder ${ }^{3}$ or of cross-sectional comparisons between first- and multiple-episode bipolar disorder showing more severe brain abnormalities in patients with multiple episodes of illness. ${ }^{4}$

On the other hand, we did find a significant decrease of total white matter volume in first-episode bipolar disorder, while Arnone et $a l^{1}$ failed to obtain the same finding in their analysis of a larger number of studies mainly conducted in patients with chronic illness. This may indicate that alterations in white matter normal growth may constitute early and primary abnormalities in bipolar disorder, consistent with some preliminary evidence of the association between patterns of disturbed structural white matter integrity in bipolar disorder and genetic liability for the illness. ${ }^{5}$ In order to explain the lack of white matter volume reduction in chronic illness, it could be hypothesised either that other, more generalised brain changes may override white matter abnormalities over the course of the disease, or that white matter changes may be attenuated by treatment or, again, may be less sensitive to the later effects of ageing. Indirect support for this idea derives from the finding of smaller volumetric differences in the temporal lobes in bipolar disorder with increasing age, duration of illness and use of mood stabilisers, ${ }^{1}$ the only discrete brain volume including white matter analysed in the meta-regressions performed by Arnone et al.

In conclusion, the finding of different brain abnormalities in chronic $v$. first-episode bipolar disorder supports the notion of different pathophysiological trajectories of specific brain morphological characteristics over the course of the disease and emphasises the need for further longitudinal studies aimed at addressing specifically the issue of the time of appearance and course of individual brain abnormalities in bipolar disorder, from which may derive a better understanding of the pathogenesis of the disease itself.

1 Arnone D, Cavanagh J, Gerber D, Lawrie SM, Ebmeier KP, McIntosh AM. Magnetic resonance imaging studies in bipolar disorder and schizophrenia: meta-analysis. Br J Psychiatry 2009; 195: 194-201.

2 Vita A, De Peri L, Sacchetti E. Gray matter, white matter, brain, and intracranial volumes in first-episode bipolar disorder: a meta-analysis of magnetic resonance imaging studies. Bipolar Disord 2009; 11: 807-14.

3 Kalmar JH, Wang $\mathrm{F}$, Spencer L, Edmiston $\mathrm{E}$, Lacadie $\mathrm{CM}$, Martin A, et al. Preliminary evidence for progressive prefrontal abnormalities in adolescents and young adults with bipolar disorder. J Int Neuropsychol Soc 2009; 15 476-81.

4 Strakowski SM, DelBello MP, Zimmerman ME, Getz GE, Mills NP, Ret J, et al. Ventricular and periventricular structural volumes in first versus multipleepisode bipolar disorder. Am J Psychiatry 2002; 159: 1841-7.

5 Chaddock CA, Barker GJ, Marshall N, schulze $\mathrm{K}$, Hall MH, et al. White matte microstructural impairments and genetic liability to familial bipolar I disorder. Br J Psychiatry 2009; 194: 527-34.

Antonio Vita, Luca De Peri, Emilio Sacchetti, University of Brescia, School of Medicine, Deparment of Mental Health, Spedali Civili Hospital, Brescia, Italy. Email: vita@med.unibs.it

doi: 10.1192/bjp.196.3.245b

Author's reply: We are grateful to Drs Vita, Peri and Sacchetti, who raise the very important point that morphometric abnormalities detectable in first-onset bipolar disorder appear different from those described in chronic patients. An observation which, as Vita et al suggest, may underpin important information about the pathogenesis of the disorder and would benefit from clarification emerging from longitudinal studies. Prompted by their meta-analysis ${ }^{1}$ and our own work, ${ }^{2}$ we have conducted further analyses by including only patients with first-episode bipolar disorder $v$. healthy controls. Despite methodological differences and different inclusion and exclusion criteria, we are in agreement with Vita et al. We found no evidence of whole-brain volume reduction in the first-episode patients $v$. healthy controls (effect size -0.23 ; $95 \%$ CI -0.47 to $0.002 ; I^{2}=0, P=0.51$; Egger's $P=0.31$ ). This finding supports Vita et als hypothesis that wholebrain volume loss may be occurring with illness progression and/ or its epiphenomena (e.g. number of episodes, pharmacological treatment). Similarly we found no evidence of gray matter loss (effect size $-0.02 ; 95 \%$ CI -0.40 to $0.37 ; I^{2}=0.02, P=0.36$; Egger's $P=0.16)$ but significant white matter volumetric reduction in the first-episode patients $v$. healthy controls (effect size $-0.45 ; 95 \% \mathrm{CI}-0.85$ to $-0.06 ; I^{2}=0.04, P=0.35$; Egger's $P=0.68$ ). These and other observations ${ }^{3,4}$ support the possibility that white matter deficits have a particular relevance to the aetiology of bipolar disorder. However, the paucity of first-episode studies is reflected in the relatively wide confidence intervals around our estimates. Further studies of patients with firstepisode bipolar disorder, as well as cohort and high-risk studies, are necessary if we are to improve our understanding of the role of structural changes in the pathogenesis of this condition.

1 Vita A, De Peri L, Sacchetti E. Gray matter, white matter, brain, and intracranial volumes in first-episode bipolar disorder: a meta-analysis of magnetic resonance imaging studies. Bipolar Disord 2009; 11: 807-14.

2 Arnone D, Cavanagh J, Gerber D, Lawrie SM, Ebmeier KP, McIntosh AM Magnetic resonance imaging studies in bipolar disorder and schizophrenia: meta-analysis. Br J Psychiatry 2009; 195: 194-201.

3 Sussmann JE, Lymer GK, McKirdy J, Moorhead TW, Maniega SM, Job D, et al. White matter abnormalities in bipolar disorder and schizophrenia detected using diffusion tensor magnetic resonance imaging. Bipolar Disord 2009; 11: $11-8$

4 McIntosh AM, Maniega SM, Lymer GK, McKirdy J, Hall J, Sussmann JE, et al. White matter tractography in bipolar disorder and schizophrenia. Biol Psychiatry 2008; 64: 1088-92.

D. Arnone, Neuroscience and Psychiatry Unit, University of Manchester, G810 Stopford Building, Oxford Road, Manchester M13 9PT, UK.

Email: danilo.arnone@manchester.ac.uk

doi: 10.1192/bjp.196.3.246

\section{Evolution and psychiatry}

If evolution is the missing half of a 'truly biological psychiatry', the other half being biological reductionism, then value is out of the picture. But this cannot be. We do not deny the gains from biology or those that are to come (millions of people manage to live because of advances in this field). Nor are we pessimistic about the potential gains that evolution claims for mental healthcare. However, these two 'halves' do not make a whole. We understand the aspiration for a truly biological psychiatry: life would be easier. Biology (although a big part, or the major part of the picture) cannot (alas!) be the whole, and evolutionary theoretical considerations of disorder, natural function, design and the like cannot fill what is missing. The reason is that even if we accept a value-free account of naturally selected mechanisms, physical as well as mental, these must be considered within the spectrum of individual and social values. Fulford ${ }^{2}$ explains why values are so feared. Other theorists who have considered 
evolution in terms of disorder also accept the indisputable place of values in psychiatry. Psychiatry is conceptually and empirically more complex than the rest of medicine. Every now and then a claim is made for a concept that will push psychiatry to an exclusively biological future. But psychiatry must be the avantgarde of science, rather than the runner-up of medicine, for perspectives which truly add to those complexities (empirical as well as philosophical) to do justice to themselves. ${ }^{3}$

Psychiatry's interest in evolutionary theory is not new. The authors will be familiar with the views of Scadding, Kendell and Boorse, who all attempted to define disease in evolutionary terms. Accounts of disorder based on evolutionary theory allegedly offered the epistemological background for a value-free conceptualisation of disease. This is one way out of psychiatry's embarrassment when comparing itself against the scientific status of the rest of medicine and the medical model. However, this seems to be a misuse of natural selection, dressed in the colours of realism in order to enhance a 'scientific' psychiatry.

We do not argue that evolutionary theory has no role to play. Evolutionary psychology may offer new significant ways of approaching mental disease. But let us hope that this interest in Darwin will not be a pretext for blind reductionism and a return to a fact/value dichotomy. Britain is in the front line of valuebased and evolution-based considerations with the work of Fulford, Thornton, ${ }^{4}$ Bolton ${ }^{5}$ and others. Great heritage, greater present.

1 Nesse, RM. Evolution at 150: time for truly biological psychiatry. $\mathrm{Br} \mathrm{J}$ Psychiatry 2009, 195: 471-2.

2 Fulford KWM, Thornton T, Graham G. Oxford Textbook of Philosophy and Psychiatry. Oxford University Press, 2006.

3 Fulford, KWM. Teleology without tears: naturalism, neo-naturalism, and evaluationism in the analysis of function statements in biology (and a bet on the twenty-first century). Philos, Psychiatry Psychol 2000; 7: 77-94.

4 Thornton T. Essential Philosophy of Psychiatry. Oxford University Press, 2007.

5 Bolton, D. Alternatives to disorder. Philos Psychiatry Psychol 2000; 7: 141-53.

Athanassios Douzenis, Dionisios Seretis, Emmanouil Rizos, loannis

Michopoulos, Christos Christodoulou, Leftaris Lykouras, Athens University

Medical School, 2nd Psychiatry Department, Attikon University Hospital, 7 Rimini

Street, Athens 12462, Greece. Email: thandouz@med.uoa.gr

doi: 10.1192/bjp.196.3.246a

Nesse $^{1}$ argues that psychiatry requires both proximate and evolutionary explanations to become a fully fledged biological science. He thinks that mental disorders such as schizophrenia and depression would benefit from posing the question of whether low mood and variable social ability were adaptive traits in times long gone and are no longer of evolutionary advantage in our current environment.

I think that Nesse's approach is as laudable as it is flawed. Evolutionary psychology proposes that most if not all human psychological traits are complex adaptations which have evolved under selective pressures. Richardson convincingly shows that the claim that all our psychological capacities have been selected for the purpose of accomplishing a particular task is too strong and that the empirical evidence required to support this claim is necessarily historical. ${ }^{2}$ The problem is, however, that the required historical evidence is hard or impossible to come by - we simply do not know what psychological traits were prevalent let alone advantageous to survive in a Pleistocene environment about which we also have little information.

For evolutionary psychology to be regarded as a credible theoretical framework it will have to be examined against standards of scientific enquiry used in other evolutionary fields such as evolutionary biology. Archaeopteryx was thought to be able to fly as it possessed feathers and claws to allow it to perch on trees. ${ }^{3}$ However, fossil records also showed that archaeopteryx lacked a sternal keel and that its awkward tail would have been likely to impede flying. Further comparative analysis showed that archaeopteryx was neither likely to perch nor be able to fly and refuted the conclusions arrived at by reverse engineering as proposed by Dennett.

Evolutionary psychology relies mainly on reverse engineering as this strategy requires comparatively few historical data but risks arriving at erroneous conclusions. Buller ${ }^{4}$ shows this to be the case for evolutionary explanations of the existence of cheater detection modules or gender differences in jealousy.

This is not to say that evolutionary psychology cannot offer an exciting and innovative framework for scientific inquiry into common mental disorders such as depression and schizophrenia but that we have to be aware of its current theoretical and methodological shortcomings and the need for further conceptual work. I agree with Geaney ${ }^{5}$ that further advances to understanding human behaviour and mental disorder would be best served by interdisciplinary cooperation whether based on evolutionary theory or not.

1 Nesse RM. Evolution at 150: time for truly biological psychiatry. Br J Psychiatry 2009; 195: 471-2.

2 Richardson RC. Evolutionary Psychology as Maladapted Psychology. MIT Press, 2007.

3 Dennett DC. Darwin's Dangerous Idea: Evolution and the Meanings of Life. Simon \& Schuster, 1995.

4 Buller DJ. Evolutionary psychology: the emperor's new paradigm. Trends Cogn Sci 2005; 9: 277-83.

5 Geaney D. Evolutionary psychiatry: the way ahead (eLetter). Br J Psychiatry 2009; http://bjp.rcpsych.org/cgi/eletters/195/6/471\#27117

Yvonne Treffurth, ST5 CAMHS, Forest House Adolescent Unit, Harperbury Hospital, Hertfordshire. Email: yvonne.treffurth@gmail.com

doi: 10.1192/bjp.196.3.247

Author's reply: Douzenis is concerned that adding evolution will make psychiatry narrowly biological in a way that excludes values. However, my article makes no claim that proximate and evolutionary approaches make up the whole of psychiatry, it says only that 'biological psychiatry is making full use of only one half of biology.' Applying this additional biological knowledge to psychiatry should not exclude values. In fact, it offers a scientific foundation for addressing the very difficulties Douzenis mentions. It is fundamentally different from 19th-century evolutionary applications to medicine. ${ }^{2}$ It is an antidote to mindless reductionism. It helps to solve the problem of defining disease, ${ }^{3}$ and to explain why psychiatric nosology is inherently problematic. ${ }^{4}$ Furthermore, profound advances in understanding human moral capacities, with important implications for psychiatry, are coming from evolutionary analyses of their origins and functions. I encourage those who share Douzenis' concerns to consider how evolutionary approaches can help us better understand our patients as individuals and provide personalised treatments that go far beyond analysing genes and prescribing drugs.

I am delighted that Treffurth finds my approach laudable, but dismayed that she seems to think my article is about evolutionary psychology. Evolutionary biology has vastly more to offer psychiatry than just evolutionary psychology, a field not mentioned in the article. I share Treffurth's concerns about the 